\title{
GENETIC DIVERSITY AND POPULATION STRUCTURE OF QUINOA (CHENOPODIUM QUINOA WILLD.) USING IPBS- RETROTRANSPOSONS MARKERS
}

\author{
Hossein-Pour, A. - Haliloglu, K. ${ }^{*}$ - OZKAn, G. - TAN, M. \\ Department of Field Crops, Faculty of Agriculture, Atatürk University, 25240 Erzurum, Turkey \\ *Corresponding author \\ e-mail: kamilh@atauni.edu.tr
}

(Received $30^{\text {th }}$ Oct 2018; accepted $16^{\text {th }}$ Jan 2019)

\begin{abstract}
Quinoa (Chenopodium quinoa Willd.) is a nutritionally important plant with a good protein quality and a high concentration of vitamins and minerals. It has been cultured for several thousand years in South America. In this study, we investigated the use of inter-primer binding site (iPBS) for the molecular characterization of 17 quinoa genotypes (Chenopodium quinoa Willd.) cultivated in Turkey. For this purpose, 25 iPBS markers were employed, and six primers provided sufficient polymorphic data generating a total of 19 alleles with an average of 2.83 bands/primer. The number of iPBS bands per individual was calculated as 1.12 . The rate of polymorphism information content ranged from 0.02 to 0.49 with an average of 0.20 . Genetic associations were assessed using the Dice dissimilarity coefficient between different pairs of accessions and revealed an average value of 0.84 for the French population and Q-52 genotypes. Cluster analysis on the unweighted pair-group mean average divided the 17 quinoa genotypes into two major clusters. The results of the principal component analysis were in agreement with those of the cluster analysis. The highest number of alleles, Nei's genetic diversity, and Shannon's information index were obtained from the French Vanilla genotype at 1.99, 0.50 and 0.69 , respectively, whereas the lowest values were observed in the Q-52 genotype at 1.10, 0.09 and 0.20 , respectively. The expected heterozygosity ranged from 0.398 in the first sub-population to 0.140 in the second subpopulation with an average of 0.269 . The mean population differentiation measurement $\left(\mathrm{F}_{\mathrm{st}}\right)$ values of the sub-populations were 0.048 and 0.676 for the first and second sub-populations, respectively. The results of this study provide useful information for the management of the quinoa germplasm and contribute to the improvement of existing breeding approaches. They also presented the iPBS marker system as a suitable tool for identification and genetic diversity analysis of quinoa genotypes.
\end{abstract}

Keywords: Bayesian clustering, Chenopodium quinoa, genetic diversity, iPBS

\section{Introduction}

In the region of Andean-South America, quinoa (Chenopodium quinoa Willd.) is considered to be one of the essential food crops. It is known as a member of the amaranth family (formerly Chenopodiaceae), which also contains other frugally essential species, such as spinach (Spinacea oleracea L.) and sugar beet (Beta vulgaris L.). Quinoa usually grows in saline and arid soils, frequent forests, and high altitudes of the Altiplano (Prado et al., 2000). Quinoa is traditionally widely consumed as a food crop by the people of the Altiplano region as part of their daily diet. Quinoa contains a perfect balance of lipids, protein, and carbohydrates, as well as amino acids essential for human nutrition (Chauhan et al., 1999). It is an allotetraploid $(2 n=4 x=36)$ which has a domestic inheritance of most quality characters (Ward, 2000).

To simplify the application of molecular tools and enhance basic knowledge concerning quinoa, Fairbanks et al. (1990) and Ruas et al. (1999) reported DNA-based markers for this species based on the random amplified polymorphic DNA (RAPD) method. Microsatellite markers (SSR) have been developed to characterize the quinoa germplasm by Mason et al. (2005), Christensen (2007), Fuentes et al. (2009), Costa 
(2012) and Lu et al. (2015). Ana-Cruz et al. (2017) and Al-Naggar et al. (2017) also attempted to characterize the genetic diversity of a collection of quinoa using intersimple sequence repeats. Employing markers, such as amplified fragment length polymorphism (AFLP), RAPD, and SSR, a genetic linkage map was established for quinoa (C. quinoa) (Maughan et al., 2004); furthermore, single nucleotide polymorphism (SNP) markers were developed (Coles et al., 2005; Maughan et al., 2012). Anabalon-Rodriguez and Thomet-Isla (2009) detected the level of polymorphism and the genetic relationships using the AFLP technique in quinoa (C. quinoa).

The fundamentally predominant existence of the tRNA complement as a converse transcriptase primer binding site (PBS) in LTR retrotransposons is a source of polymerase chain reaction (PCR)-based iPBS amplification (inter-primer binding site iPBS). Specifically, the iPBS amplification technique was developed as a remarkable DNA fingerprinting technology that does not require any primary sequence data. Therefore, for controlling the changes in the DNA profile of plants, the iPBS marker system is an easy and fast process. This technique has been successfully used in flax (Smykal et al., 2011), apricot (Baránek et al., 2012), latvia (Saussurea esthonica L.) (Gailite and Rungis, 2012), chickpea (Andeden et al., 2013), guava (Mehmood et al., 2016), grape (Guo et al., 2014), okra (Yildiz et al., 2015), rice (Comertpay et al., 2015), lentil and pea (Baloch et al., 2015), tea (Phong et al., 2016), saffron (Gedik et al., 2017) and motherwort (Leonurus cardiaca L.) (Borna et al., 2017). In this study, to simplify the application of molecular tools and offer a better understanding of the genetic diversity of quinoa genotypes, we utilized iPBS molecular markers for these species for the first time in the literature.

The purpose of this experiment was to investigate the genetic diversity of 17 quinoas genotypes using the iPBS marker system, to evaluate the structure of the diversity in the germplasm, and to generate useful information for future breeding programs on quinoa.

\section{Materials and methods}

\section{Genetic material}

The quinoa (C. quinoa Willd.) genotypes were collected from different countries as a work package of a project supported by The Scientific and Technological Research Council of Turkey (TUBITAK) (TOVAG 214 O 232). A total of 17 quinoas genotypes were used in the experiment (Table 1).

\section{Genomic DNA isolation}

Total genomic DNA from the quinoa genotypes was isolated from $300 \mathrm{mg}$ young leaf tissue using the method described by Zeinalzadeh-Tabrizi et al. (2015). For determination of the concentration and quality of genomic DNA, a spectrophotometer (Thermo Fisher Scientific) was used and electrophoresis was performed in $0.8 \%(\mathrm{w} / \mathrm{v})$ agarose gel.

\section{iPBS-PCR amplification}

Twenty-five primers were used in iPBS-PCR reactions (Table 2). The PCR amplifications were carried out in a thermal cycler (Labcycler). The PCR mixture consisted of $10 \mathrm{x}$ buffer, $2 \mu \mathrm{M}$ (20 pmol) primer, $2 \mathrm{mM} \mathrm{MgCl} 2,0.25 \mathrm{mM}$ of each dNTP, $0.5 \mathrm{U} \mathrm{Taq}$ polymerase, and $50 \mathrm{ng} / \mu \mathrm{l}$ DNA template in a total volume of $20 \mu \mathrm{l}$. The 
amplification conditions were; an initial denaturation step of five min at $95{ }^{\circ} \mathrm{C}, 38$ cycles of $60 \mathrm{~s}$ at $95{ }^{\circ} \mathrm{C}, 60 \mathrm{~s}$ at $44-60{ }^{\circ} \mathrm{C}$ and $60 \mathrm{~s}$ at $72{ }^{\circ} \mathrm{C}$, and a final extension step of $10 \mathrm{~min}$ at $72{ }^{\circ} \mathrm{C}$. The amplification products were resolved in $1.5 \%$ agarose gel in $1 \mathrm{X}$ SB buffer at $100 \mathrm{~V} / \mathrm{cm}$ for $120 \mathrm{~min}$, stained with ethidium bromide $(0.2 \mathrm{ug} / \mathrm{ml})$, and visualized under a UV light in a Universal Hood II (Bio-Rad, Hercules, CA, USA). The sizes of the base pairs were determined based on a DNA ladder between 50 and $1000 \mathrm{bp}$ (Vivantis Product No: NM2421).

Table 1. List of quinoa (Chenopodium quinoa Willd.) accessions used in the experiment

\begin{tabular}{c|c|c}
\hline Number & Genotype name & Country \\
\hline 1 & Ecuador 7 & Ecuador \\
2 & Q haslala Blanca & Peru \\
3 & Red population & Peru \\
4 & Q-52 & Denmark \\
5 & White Population & United Kingdom \\
6 & Titicaca & Peru \\
7 & UK6 & USA \\
8 & French Vanilla & USA \\
9 & Red Head & USA \\
10 & Sandoval Mix & United Kingdom \\
11 & Mint Valle & USA \\
12 & Oro de Valle & USA \\
13 & Chinese Population & China \\
14 & France Population & France \\
15 & Chery Vanilla & USA \\
16 & Moqu Arrochilla & Peru \\
17 & Rainbow & USA \\
\hline
\end{tabular}

\section{Data analysis}

For each primer, the presence and absence of a strong and sharp polymorphic band were scored as 1 and 0 , respectively using TotalLab TL120 software package (Germany). The association between the genetic dissimilarity was evaluated with the Numerical Taxonomy and Multiware Analysis System (NTSYSpc version 2.0) according to the Dice similarity matrix (Dice, 1945). Using the same software, an unweighted pair-group mean average (UPGMA) tree was constructed and a principle component analysis (PCA) was undertaken (Rohlf, 1998). The diversity of each iPBS marker was calculated using polymorphism information content (PIC) according to the following equation: $P I C=1-\Sigma p i 2$, where $P_{i j}$ is the frequency of the patterns $(j)$ for each marker (i) (Anderson et al., 1993). To determine the genetic parameters, the number of alleles (ne), Nei's genetic diversity (h), and Shannon's information index (I) were calculated by POPGEN 1.32 (Yeh et al., 1997).

The genetic structure datasets were formed to be determined by a model-based cluster analysis using STRUCTURE software version 2.2 (Pritchard et al., 2000a, b). The number of populations (K) was presented for a run of ten times for each population, which varied from 2 to 10 , characterized by a set of distinctive allele frequencies at each locus, and individuals were situated in $\mathrm{K}$ clusters. In this process, the posterior 
probabilities were estimated using the Markov Chain Monte Carlo (MCMC) method. The MCMC chains were run with a 100,000-iteration burn-in period, followed by 100.000 iterations using a model allowing for admixture and correlated allele frequencies. The most expected value for $\mathrm{K}$ was predicted using Evanno's $\Delta \mathrm{K}$ method (Evanno et al., 2005) using STRUCTURE HARVESTER. Furthermore, the expected heterozygosity (gene diversity) and population differentiation (Fst) between the individuals in a sub-population were determined using STRUCTURE (Earl, 2012).

Table 2. Primers used in iPBS -PCR and retrotransposon, amplified fragment length

\begin{tabular}{c|c|c}
\hline Primer no. & Primer name & Primer sequence $\left(\mathbf{5}^{\prime} \rightarrow \mathbf{3}^{\prime}\right)$ \\
\hline 1 & iPBS-2074 & GCTCTGATACCA \\
2 & iPBS-2075 & CTCATGATGCCA \\
3 & iPBS-2076 & GCTCCGATGCCA \\
4 & iPBS-2077 & CTCACGATGCCA \\
5 & iPBS-2080 & CAGACGGCGCCA \\
6 & iPBS-2221 & ACCTAGCTCACGATGCCA \\
7 & iPBS-2222 & ACTTGGATGCCGATACCA \\
8 & iPBS-2224 & ATCCTGGCAATGGAACCA \\
9 & iPBS-2225 & AGCATAGCTTTGATACCA \\
10 & iPBS-2270 & ACCTGGCGTGCCA \\
11 & iPBS-2279 & AATGAAAGCACCA \\
12 & iPBS-2375 & TCGCATCAACCA \\
13 & iPBS-2376 & TAGATGGCACCA \\
14 & iPBS-2377 & ACGAAGGGACCA \\
15 & iPBS-2378 & GGTCCTCATCCA \\
16 & iPBS-2379 & TCCAGAGATCCA \\
17 & iPBS-2380 & CAACCTGATCCA \\
18 & iPBS-2381 & GTCCATCTTCCA \\
19 & iPBS-2390 & GCAACAACCCCA \\
20 & iPBS-2391 & ATCTGTCAGCCA \\
21 & iPBS-2392 & TAGATGGTGCCA \\
22 & iPBS-2400 & CCCCTCCTTCTAGCGCCA \\
23 & iPBS-2401 & AGTTAAGCTTTGATACCA \\
24 & iPBS-2402 & CATCGTAGGTGGGCGCCA \\
25 & iPBS-2415 & \\
\hline
\end{tabular}

\section{Results and discussion}

\section{Polymorphism revealed by iPBS primers}

In this experiment, $25 \mathrm{iPBS}$ primers were used to evaluate 17 quinoa genotypes, and only six primers generated a sufficient number of polymorphisms (Fig. 1) with four primers providing more than one polymorphic band. The average of polymorphic bands was calculated as 2.83 , and that of monomorphic bands was 0.33. Based on these values, the number of iPBS bands per individual was calculated as 1.12 (Table 3). 


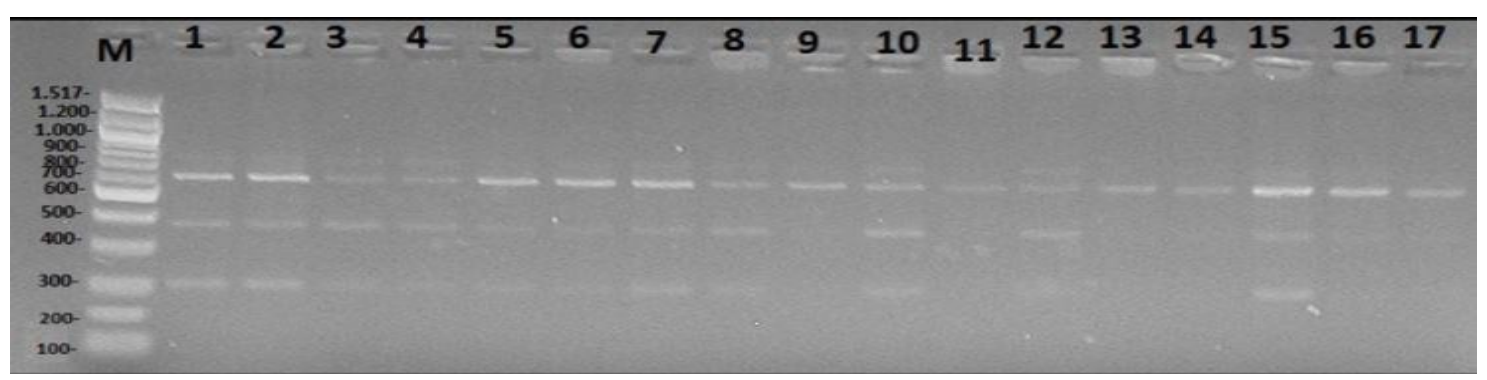

Figure 1. iPBS profiles of 17 quinoa accessions obtained with the primer iPBS 2391

Table 3. Diversity statistics for Quinoa (Chenopodium quinoa Willd.) with iPBS

\begin{tabular}{c|c|c|c}
\hline Primer name & Allele number & Percentage of polymorphism & PIC value \\
\hline iPBS-2080 & 7 & $100.00 \%$ & 0.49 \\
iPBS-2270 & 1 & $100.00 \%$ & 0.03 \\
iPBS-2279 & 3 & $66.66 \%$ & 0.14 \\
iPBS-2390 & 1 & $100.00 \%$ & 0.02 \\
iPBS-2391 & 4 & $75.00 \%$ & 0.23 \\
iPBS-2392 & 3 & $100.00 \%$ & 0.18 \\
\hline
\end{tabular}

Similar to our results, Mehmood et al. (2013) selected 6 primers out of 83 iPBS primer set in guava genotypes and Guo et al. (2014) selected 15 primers out of 41 iPBS primers in grape varieties, for further analysis. This is also in agreement with reports on other plants, such as grape (Guo et al., 2014), guava (Mehmood et al., 2016), and those investigated by Kalendar et al. (2010). The total number of polymorphic bands was 19 for iPBS markers, of which 17 were polymorphic and the remainder were considered as monomorphic. In this research, the number of alleles/polymorphic loci ranged from one to seven with an average of 3.16 for the iPBS analysis. Furthermore, the highest number of polymorphisms was obtained from iPBS-2080 with seven bands. In a study that aimed to develop molecular markers for the Fusarium wilt resistance gene in eggplant, Mutlu et al. (2008) found an average number of 1.5 bands per primer. The PIC value varied between 0.02 (iPBS 2390) and 0.49 (iPBS 2080), with an average of 0.20 (Table 3). These results are in agreement with earlier research into different plants, such as guava (Psidium guajava L.) cultivars with an average PIC of 0.28 (Mehmood et al., 2016) and tea (Camellia sinensis) cultivars with an average PIC of 0.30 (Phong et al., 2016). The percentage of polymorphism (\%) value ranged from 66\% (iPBS 2279) to 100\% (iPBS 2080, 2390 and 2392) with an average of 90.27\% (Table 3).

\section{Genetic diversity in quinoa}

Table 4 presents a summary of the statistical results for each of the 17 quinoa genotypes. The highest number of alleles (ne), Nei's genetic diversity (h), and Shannon's information index (I) were obtained from the French Vanilla genotype at $1.99,0.50$ and 0.69 , respectively, whereas the lowest values were observed in the Q-52 genotype at 1.10, 0.09 and 0.20, respectively. In addition, the total average number of alleles (ne), Nei's genetic diversity (h), and Shannon's information index (I) were 1.52, 0.032 and 0.49, respectively. Fuentes et al. (2009) used 20 SSR loci and calculated 
Shannon's index as 2.582 for the Highland genotype and 3.714 for the coastal genotypes Furthermore, the average value of Shannon's information index was reported as 0.12 in the Turkish okra germplasm (Yildiz et al., 2015), 0.29 for the Crocus sativus genotypes (Gedik et al., 2017), and 0.27 for guava (Mehmood et al., 2013) using iPBSretrotransposon markers.

Table 4. Summary statistics for 17 for Quinoa genotypes assessed with 10 iPBS primers

\begin{tabular}{c|c|c|c|c}
\hline Number & Genotype name & (ne) $^{*}$ & (h) & (I) \\
\hline 1 & Ecuador 7 & 1.36 & 0.27 & 0.44 \\
2 & Q haslala Blanca & 1.63 & 0.39 & 0.58 \\
3 & Red Population & 1.63 & 0.39 & 0.58 \\
4 & Q - 52 & 1.10 & 0.09 & 0.20 \\
5 & White Population & 1.63 & 0.39 & 0.58 \\
6 & Titicaca & 1.23 & 0.19 & 0.34 \\
7 & UK6 & 1.23 & 0.19 & 0.34 \\
8 & French Vanilla & 1.99 & 0.50 & 0.69 \\
9 & Red Head & 1.50 & 0.33 & 0.51 \\
10 & Sandoval Mix & 1.11 & 0.10 & 0.21 \\
11 & Mint Valle & 1.50 & 0.33 & 0.51 \\
12 & Oro de Valle & 1.36 & 0.27 & 0.44 \\
13 & Chinese Population & 1.95 & 0.49 & 0.68 \\
14 & France Population & 1.36 & 0.27 & 0.44 \\
15 & Chery Vanilla & 1.50 & 0.33 & 0.51 \\
16 & Moqu Arrochilla & 1.87 & 0.47 & 0.66 \\
17 & Rainbow & 1.87 & 0.47 & 0.66 \\
\hline \multicolumn{4}{l|}{}
\end{tabular}

$*_{\text {ne }}=$ Effective number of alleles, $\mathrm{h}=$ Nei gene diversity, $\mathrm{I}=$ Shanon information index

\section{Cluster analysis and principal component analysis for iPBS-retrotransposon markers}

The Dice genetic similarity coefficient was used for the diversity estimation of the genotypes. This coefficient is commonly utilized to estimate genetic distances. The genetic similarity between the accessions based on the iPBS markers ranged from $11 \%$ to $100 \%$ with an average value of $55.5 \%$. In this study, the $100 \%$ similarity value was found between the two most closely related accessions, Titicaca and UK6, and Q haslala Blanca and Red (Table 5). Nemli et al. (2014) reported similar findings in a study that explored the genetic similarity between the accessions of common bean (Phaseolus vulgaris L.) by peroxidase gene-based markers. The authors found the similarity coefficients to vary between 0.7 and 1 .

The UPGMA tree constructed using the Jaccard genetic distance coefficient is presented in Figure 2. The analysis divided the quinoa genotypes into two main groups: Group A containing three genotypes (17.65\%) and group B containing 14 genotypes $(82.35 \%)$. Group A had two sub-groups: the first containing French Population and the second comprising the genotypes Chinese Population and Mint Valle. Similar to group A, group B had two sub-groups; the first containing Rainbow, Moqu Arochilla, and Red Head and the second comprising French Vanilla, USA 4, Red Population, Q Blanca, Sandoval Mix, Q-52, Chery Vanilla, Oro de Volle, UK6, Titicaca, and Ikwadur 7 (Fig. 2). 
Table 5. Dice genetic similarity coefficient among 17 quinoas genotypes based iPBS

\begin{tabular}{l|c|c|c|c|c|c|c|c|c|c|c|c|c|c|c|c|c}
\hline & $(\mathbf{1})$ & $\mathbf{( 2 )}$ & $\mathbf{( 3 )}$ & $\mathbf{( 4 )}$ & $\mathbf{( 5 )}$ & $\mathbf{( 6 )}$ & $\mathbf{( 7 )}$ & $\mathbf{( 8 )}$ & $\mathbf{( 9 )}$ & $\mathbf{( 1 0 )}$ & $\mathbf{( 1 1})$ & $\mathbf{( 1 2})$ & $\mathbf{( 1 3 )}$ & $\mathbf{( 1 4 )}$ & $\mathbf{( 1 5 )}$ & $\mathbf{( 1 6 )}$ & $\mathbf{( 1 7 )}$ \\
\hline (1) Ecuador 7 & 1 & & & & & & & & & & & & & & & & \\
\hline (2) Qhaslala Blanca & 0.11 & 1 & & & & & & & & & & & & & & & \\
\hline (3) Red Population & 0.11 & 1.00 & 1 & & & & & & & & & & & & & & \\
\hline (4) Q-52 & 0.16 & 0.26 & 0.26 & 1 & & & & & & & & & & & & & \\
\hline (5) White Population & 0.11 & 0.21 & 0.21 & 0.26 & 1 & & & & & & & & & & & & \\
\hline (6) Titicaca & 0.05 & 0.16 & 0.16 & 0.11 & 0.16 & 1 & & & & & & & & & & & \\
\hline (7) UK6 & 0.05 & 0.16 & 0.16 & 0.11 & 0.16 & 1.00 & 1 & & & & & & & & & & \\
\hline (8) French Vanilla & 0.32 & 0.21 & 0.21 & 0.47 & 0.32 & 0.37 & 0.37 & 1 & & & & & & & & & \\
\hline (9) Red Head & 0.37 & 0.47 & 0.47 & 0.21 & 0.37 & 0.32 & 0.32 & 0.58 & 1 & & & & & & & & \\
\hline (10) Sandoval Mix & 0.11 & 0.21 & 0.21 & 0.05 & 0.21 & 0.16 & 0.16 & 0.42 & 0.26 & 1 & & & & & & & \\
\hline (11) Mint Valle & 0.63 & 0.53 & 0.53 & 0.79 & 0.53 & 0.68 & 0.68 & 0.32 & 0.58 & 0.74 & 1 & & & & & & \\
\hline (12) Oro de Valle & 0.11 & 0.21 & 0.21 & 0.16 & 0.21 & 0.05 & 0.05 & 0.42 & 0.37 & 0.21 & 0.63 & 1 & & & & & \\
\hline (13) Chinese Population & 0.42 & 0.42 & 0.42 & 0.58 & 0.32 & 0.47 & 0.47 & 0.32 & 0.37 & 0.53 & 0.21 & 0.42 & 1 & & & & \\
\hline (14) France Population & 0.68 & 0.58 & 0.58 & 0.84 & 0.58 & 0.74 & 0.74 & 0.37 & 0.63 & 0.79 & 0.16 & 0.68 & 0.26 & 1 & & & \\
\hline (15) Chery Vanilla & 0.16 & 0.26 & 0.26 & 0.21 & 0.26 & 0.11 & 0.11 & 0.47 & 0.42 & 0.26 & 0.68 & 0.05 & 0.47 & 0.63 & 1 & & \\
\hline (16) Moqu Arrochilla & 0.42 & 0.53 & 0.53 & 0.37 & 0.42 & 0.47 & 0.47 & 0.63 & 0.26 & 0.32 & 0.53 & 0.42 & 0.32 & 0.47 & 0.37 & 1 & \\
\hline (17) Rainbow & 0.53 & 0.63 & 0.63 & 0.37 & 0.53 & 0.47 & 0.47 & 0.74 & 0.26 & 0.42 & 0.63 & 0.42 & 0.42 & 0.47 & 0.37 & 0.11 & 1 \\
\hline
\end{tabular}

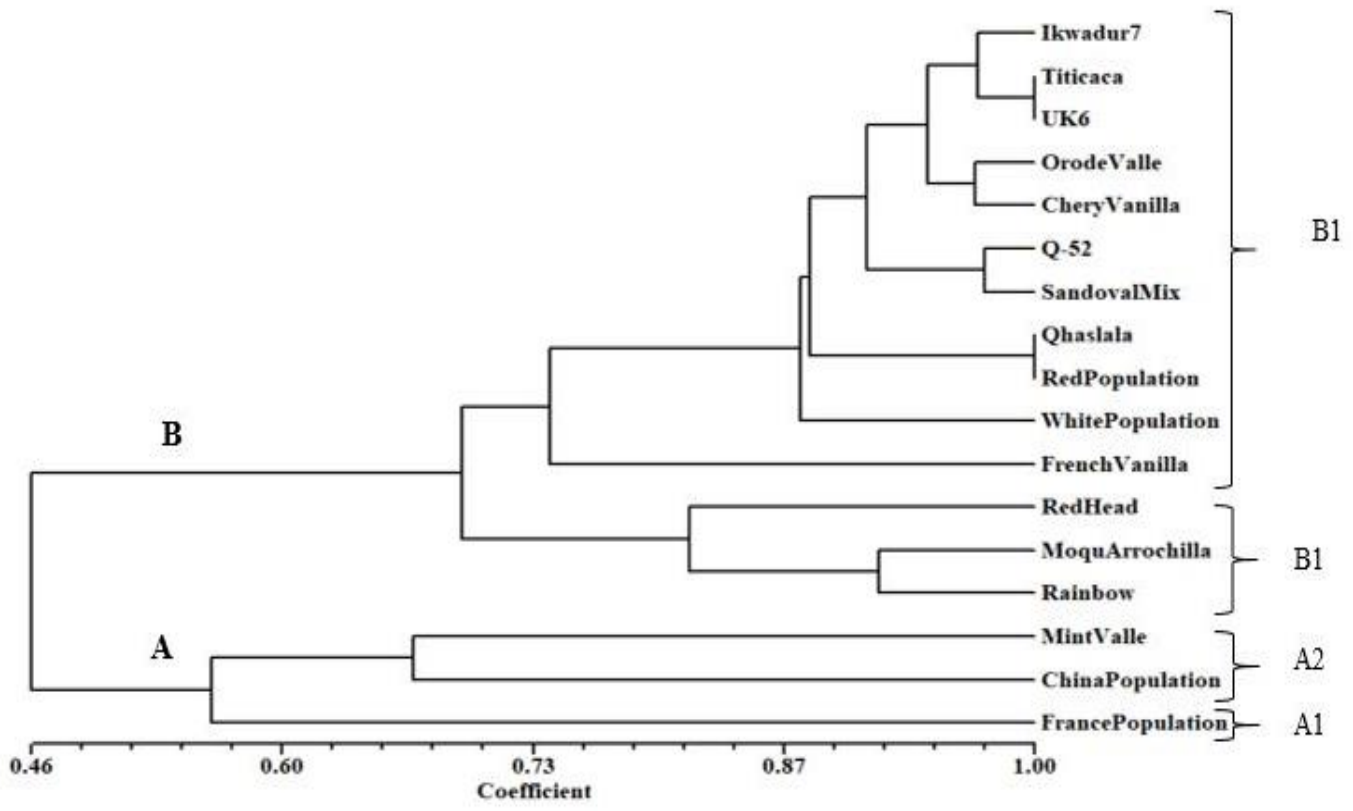

Figure 2. Dendrogram of 17 Quinoa genotypes based on iPBS markers according to UPGMA with the Dice similarity index

As a one-dimensional reduction technique, PCA can be used to review molecular marker profiles into some uncorrelated components (Hotelling, 1933). The pattern of cluster analysis was further confirmed by PCA because this method provides a betterdefined structure than a dendrogram. In the current study, PCA was used to determine the variations between the two populations (Fig. 3). The clustering of varieties based on a dendrogram and a PCA plot was similar with no discrepancy being observed. This result is supported by Belaj et al. (2002) and Zargar et al. (2014), who reported similar 
findings using RAPD and SSR markers obtained similar findings related to the diversity of 32 olive cultivars based on the dendrogram topologies.

Christensen et al. (2007) evaluated the hereditary mutation in the USDA and CIPFAO international nursery collections of quinoa (Chenopodium quinoa Willd.) by means of microsatellite markers and found that both UPGMA and PCA analyses divided the quinoa accessions into two key groups. Fuentes et al. (2009) investigated the genetic variety patterns in the Chilean quinoa (Chenopodium quinoa Willd.) germplasm using multiplex fluorescent microsatellite markers and reported that PCA divided PC1 into two major branches, conforming the clusters and groups of the highland and coastal quinoa accessions of the UPGMA analysis.

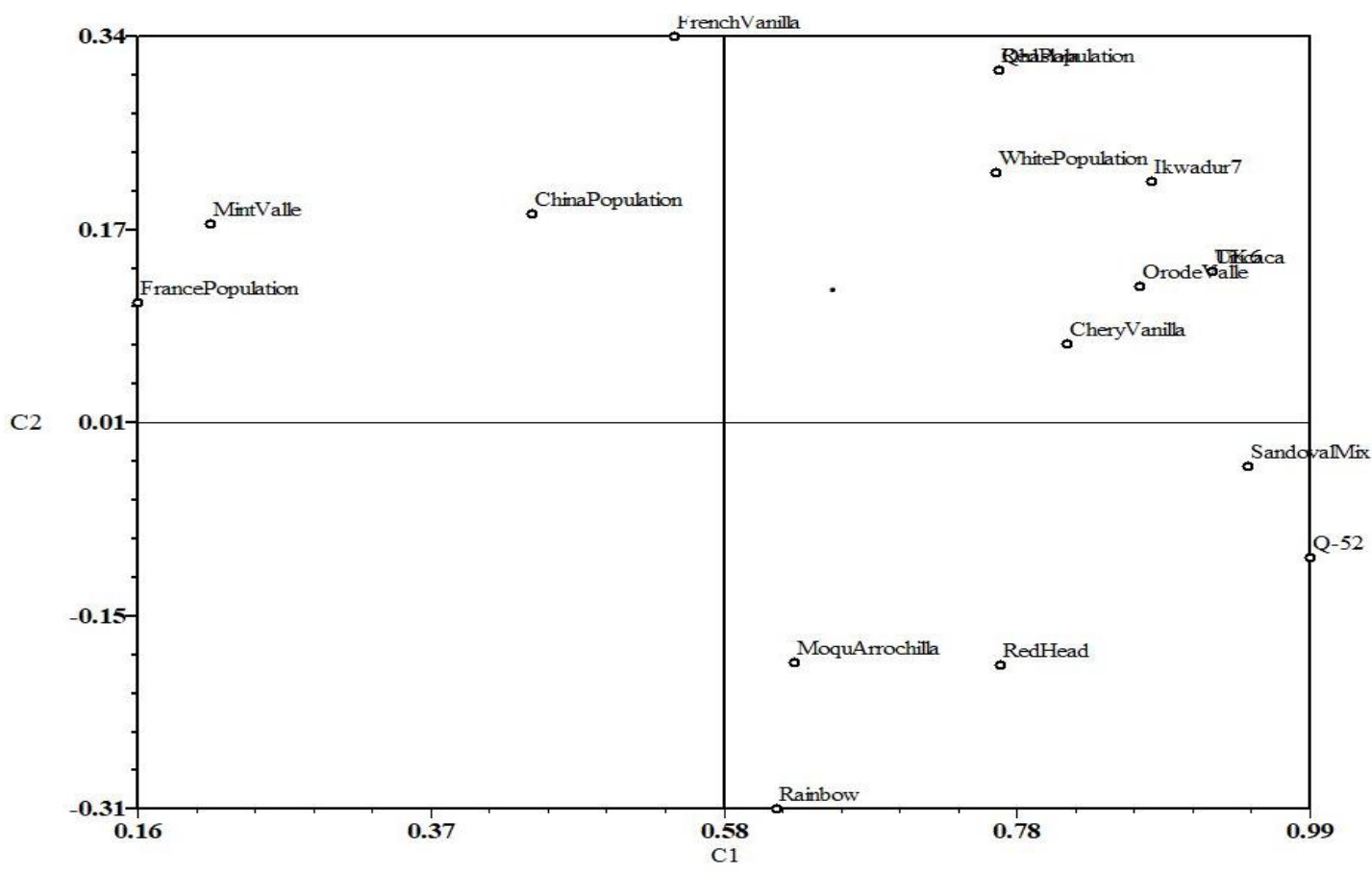

Figure 3. PCA of 17 Quinoa genotypes based on 10 iPBS markers

\section{Population genetic structure analysis for iPBS-retrotransposon markers}

Crop improvement is based on the understanding of the population assembly of germplasm collections. Before performing an association mapping study, it is crucial to first set the population structure within the germplasm to avoid spurious associations (Flint-Garcia et al., 2005). In this research, the population structure of the 17 quinoa accessions was categorized according to the iPBS data using STRUCTURE version 2.2 (Fig. 4) and two sub populations were found. Population 1 (POP 1) contained three accessions (French Population, Chinese Population and Mint Valle) and Population 2 (POP 2) consisted of the following 14 accessions (82.35\% with membership probability of $<0.7$ ): Rainbow, Moqu Arochilla, Red Head, French Vanilla, USA 4, Red Population, Q Blanca, Sandoval Mix, Q-52, Chery Vanilla, Oro de Volle, UK6, Titicaca, and Ikwadur 7). The membership coefficient of the genotypes to specific sub-populations was very high and no possible admixture was detected in a reduced number of landraces. This may be due to the high rate of self-pollination of quinoa. Similar results were reported by Zhang et al. (2017) investigating the development of novel InDel 
markers and genetic diversity in Chenopodium quinoa through whole-genome resequencing. The authors found that according to both $L(K)$ and $\triangle K$ values, the two groups presented the optimal classification for these quinoa accessions. Parallel results were reported with high degrees of genetic variation as detected by AFLP in Sideritis tmolea by Nemli et al. (2014), who revealed the presence of a model-based structural analysis of two populations. Yoon et al. (2012) reported genetic diversity and population structure analysis of two populations of strawberry (Fragaria $\mathrm{x}$ ananassa Duch.) using SSR markers.

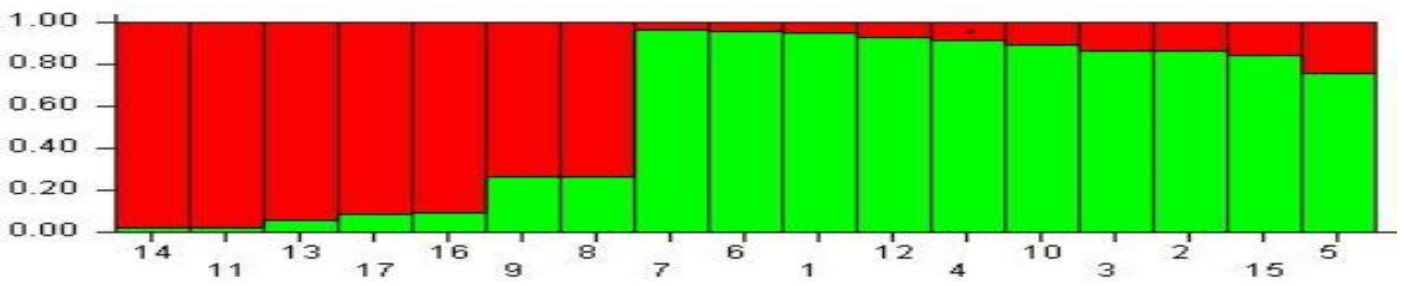

Figure 4. Genetic structure of 17 quinoas genotypes as inferred by STRUCTURE software with 10 iPBS marker data sets. Single vertical line represents an individual accession and different colors represent genetic stocks/gene pools. Segments of each vertical line show extent of admixture in an individual

In the current study, the population structure analysis confirmed the grouping of the genotypes, as observed by PCA and UPGMA clustering analyses. Similarly, Chen et al. (2015), who developed SSR markers to assess the genetic diversity of adzuki bean in the Chinese germplasm collection, showed that the structure and cluster analyses were usually consistent.

These results support the idea that dissimilar elevations in a topographical area might result in various levels of selection pressure for modification and could increase the differences within a population (Lopez-Gartner et al., 2009). The variation between gene pools suggests that cross breeding among these diverse areas will accelerate the process of diversifying germplasm creation and widen germplasm resources of quinoa. Meanwhile, efforts are being made to gather samples from different regions and produce the most effective markers to clarify the genetic diversity, population structure, and other details of population changeability in this ergonomically important genotype.

The expected heterozygosity which measures the probability of two randomly chosen individuals being different (heterozygous) in a given locus ranged from 0.398 in population 1 to 0.140 in the population 2 with an average of 0.269 . The mean population differentiation measurement $\left(\mathrm{F}_{\text {st }}\right)$ values of the sub-populations were 0.048 and 0.676 (Table 6) for the first and second sub-populations, which was relatively high confirming the separation of the two sub-populations and their diversity in iPBS alleles. Similar results were reported by Zargar et al. (2016) who found that according to the population differentiation measurements (Fst), there were two distinct clusters or populations with an average Fst of 0.3301 , indicating a clear separation of the subpopulations and their diversity in RAPD and SSR alleles. Blair et al. (2012) analyzed 108 common bean genotypes using 36 fluorescently labeled SSRs and also observed a high Fst value $(0.203)$ for the genetic differentiation between all the five populations. In the current study, we obtained an even higher Fst value as a result of using the iPBS marker system. 
Table 6. Heterozygosity and Fst value calculated for 2 quinoas sub-populations

\begin{tabular}{c|c|c}
\hline Sub-population (K) & Expected heterozygosity & Fst value \\
\hline 1 & 0.398 & 0.048 \\
2 & 0.140 & 0.676 \\
Average & 0.269 & 0.362 \\
\hline
\end{tabular}

\section{Conclusion}

Molecular markers are effectively used to explore genetic variation to enhance breeding efficiency. This study was undertaken using iPBS molecular markers for quinoa genotypes to simplify the application of this method and provide essential data on these genotypes. We also effectively categorized the population structure of 17 quinoa genotypes cultivated in Turkey using iPBS markers and model-based clustering. Moreover, the data obtained from the population structure analysis is valuable to perform association mapping on quinoa genotypes for various traits. The results obtained during the study can assist in the decision-making process concerning the selecting of markers for future experiments, as well as further characterization, breeding and management of the quinoa germplasm.

Acknowledgements. We greatly appreciate our grant from the Scientific and Technological Research Council of Turkey for project "TUBITAK 214 O 232".

\section{REFERENCES}

[1] Al-Naggar, A. M. M., El-Salam, R. A., Badran, A. E. E. and El-Moghazi, M. M. (2017): Molecular differentiation of five Quinoa (Chenopodium quinoa Willd.) genotypes using inter-simple sequence repeat (ISSR) markers. - British Biotechnology Journal 20: 1-12.

[2] Anabalo'n-Rodri'guez, L., Thomet-Isla, M. (2009): Comparative analysis of genetic and morphologic diversity among quinoa accessions (Chenopodium quinoa Willd.) of the South of Chile and highland accessions. - Journal of Plant Breeding and Crop Science 1: 210-216.

[3] Ana-Cruz, M. C., Manjarres, E. H., Yacenia, M. C. (2017): Molecular characterization of Chenopodium quinoa Willd. using inter-simple sequence repeat (ISSR) markers. African Journal of Biotechnology 16: 483.

[4] Andeden, E. E., Baloch, F. S., Derya, M., Kilian, B., Özkan, H. (2013): iPBSRetrotransposons-based genetic diversity and relationship among wild annual Cicer species. - Journal of Plant Biochemistry and Biotechnology 22: 453-466.

[5] Anderson, J. A., Churchill, G. A., Autrique, J. E., Tanksley, S. D., Sorrells, M. E. (1993): Optimizing parental selection for genetic-linkage maps. - Genome 36: 181-186.

[6] Baloch, F. S., Alsaleh, A., de Miera, L. E. S., Hatipoğlu, R., Çiftçi, V., Karaköy, T., Yıldız, M., Özkan, H. (2015): DNA based iPBS-retrotransposon markers for investigating the population structure of pea (Pisum sativum) germplasm from Turkey. - Biochemical Systematics and Ecology 61: 244-252.

[7] Baránek, M., Meszáros, M., Sochorová, J., Čechová, J., Raddová, J. (2012): Utility of retrotransposon-derived marker systems for differentiation of presumed clones of the apricot cultivar Velkopavlovická. - Scientia Horticulturae 143: 1-6.

[8] Belaj, A., Satovic, Z., Rallo, L., Trujillo, I. (2002): Genetic diversity and relationship in olive (Olea europaea L.) germplasm collections as determined by randomly amplified polymorphic DNA. - Theoretical and Applied Genetics 105: 638-644. 
[9] Blair, M. W., Soler, A., Corte, A. J. (2012): Diversification and population structure in common beans (Phaseolus vulgaris L.). - PLoS ONE 7: 11 e49488.

[10] Borna, F., Luo, S., Ahmad, N. M., Nazeri, V., Shokrpour, M., Trethowan, R. (2017): Genetic diversity in populations of the medicinal plant Leonurus cardiaca L. revealed by inter-primer binding site (iPBS) markers. - Genetic Resources and Crop Evolution 64: 479-492.

[11] Chauhan, G. S., Eskin, N. A. M., Tkachuk, R. (1999): Effect of saponin extraction on quality of quinoa (Chenopodium quinoa Willd.). - Journal of Food Science and Technology 36: 123-126.

[12] Chen, H., Liu, L., Wang, L., Wang, S., Wang, M. L., Cheng, X. (2015): Development of SSR markers and assessment of genetic diversity of adzuki bean in the Chinese germplasm collection. - Molecular Breeding 35: 191.

[13] Christensen, S. A., Pratt, D. B., Pratt, C., Nelson, P. T., Stevens, M. R., Jellen, E. N., Maughan, P. J. (2007): Assessment of genetic diversity in the USDA and CIP-FAO international nursery collections of quinoa (Chenopodium quinoa Willd.) using microsatellite markers. - Plant Genetic Resources 5: 82-95.

[14] Coles, N. D., Coleman, C. E., Christensen, S. A., Jellen, E. N., Stevens, M. R., Bonifacio, A., Rojas-Beltran, J. A., Fairbanks, D. J., Maughan, P. J. (2005): Development and use of an expressed sequenced tag library in quinoa (Chenopodium quinoa Willd.) for the discovery of single nucleotide polymorphisms. - Plant Science 168: 439-447.

[15] Comertpay, G., Baloch, F. S., Derya, M., Andeden, E. E., Alsaleh, A., Surek, H., Ozkan, H. (2015): Population structure of rice varieties used in Turkish rice breeding programs determined using simple-sequence repeat and inter-primer binding site-retrotransposon data. - Genetics and Molecular Research 15: 1.

[16] Costa-Ta'rtara, S. M., Manifesto, M. M., Bramardi, S. J., Bertero, H. D. (2012): Genetic structure in cultivated quinoa (Chenopodium quinoa Willd.), a reflection of landscape structure in Northwest Argentina. - Conservation Genetics 13: 1027-1038.

[17] Dice, L. R. (1945): Measures of the amount of ecologic association between species. Ecosphere 26: 297-302.

[18] Earl, D. A. (2012): STRUCTURE HARVESTER: a website and program for visualizing STRUCTURE output and implementing the Evanno method. - Conservation Genetics Resources 4: 359-361.

[19] Evanno, G., Regnaut, S., Goudet, J. (2005): Detecting the number of clusters of individuals using the software STRUCTURE: a simulation study. - Molecular Ecology 14: 2611-2620.

[20] Fairbanks, D. J., Burgener, K. W., Robison, L. R., Andersen, W. R., Ballon, E. (1990): Electrophoretic characterization of quinoa seed proteins. - Plant breeding 104: 190-195.

[21] Flint-Garcia, S. A., Thuillet, A. C., Yu, J., Pressoir, G., Romero, S. M., Mitchell, S. E., Doebley, J., Kresovich, S., Goodman, M. M., Buckler, E. S. (2005): Maize association population: a high-resolution platform for quantitative trait locus dissection. - Plant Journal 44: 1054-1064.

[22] Fuentes, F. F., Martı'nez, E., Hinrichsen, P., Jellen, E., Maughan, P. (2009): Assessment of genetic diversity patterns in Chilean quinoa (Chenopodium quinoa Willd.) germplasm using multiplex fluorescent microsatellite markers. - Conservation Genetics 10: 369-377.

[23] Gailite, A., Rungis, D. (2012): An initial investigation of the taxonomic status of Saussurea esthonica Baer ex Rupr. utilising DNA markers and sequencing. - Plant Systematics and Evolution 298: 913-919.

[24] Gedik, A., Ates, D., Erdogmus, S., Comertpay, G., Bahattin, M. (2017): Genetic diversity of crocus sativus and its close relative species analyzed by ipbs-retrotransposons. Turkish Journal of Field Crops 22: 243-252.

[25] Guo, D. L., Guo, M. X., Hou, X. G., Zhang, G. H. (2014): Molecular diversity analysis of grape varieties based on iPBS markers. - Biochemical Systematics and Ecology 52: 2732. 
[26] Hotelling, H. (1933): Analysis of a complex of statistical variables into principal components. - Journal of Educational Psychology 24: 417-441.

[27] Kalendar, R., Antonius, K., Smýkal, P., Schulman, A. H. (2010): iPBS: a universal method for DNA fingerprinting and retrotransposon isolation. - Theoretical and Applied Genetics 121: 1419-1430.

[28] Lopez-Gartner, G., Cortina, H., McCouch, S. R., Moncada, M. D. (2009): Analysis of genetic structure in a sample of coffee (Coffea arabica L.) using fluorescent SSR markers. - Tree Genetics and Genomes 5: 435-446.

[29] Lu, M., Mo, X., Wang, Q., Lu, G., Jiang, Y. (2015): Comparison of genomic DNA extraction methods for Chenopodium quinoa Willd. - Agricultural Science and Technology 6: 1343.

[30] Mason, S. L., Stevens, M. R., Jellen, E. N., Bonifacio, A., Fairbanks, D. J., Coleman, C. E., McCarty, R. R., Rasmussen, A. G., Maughan, P. J. (2005): Development and use of microsatellite markers for germplasm characterization in quinoa (Chenopodium quinoa Willd.). - Crop Science 45: 1618-1630.

[31] Maughan, P. J., Bonifacio, A., Jellen, E. N., Stevens, M. R., Coleman, C. E., Ricks, M., Mason, S. L., Jarvis, D. E., Gardunia, B. W., Fairbanks, D. J. (2004): A genetic linkage map of quinoa (Chenopodium quinoa) based on AFLP, RAPD, and SSR markers. Theoretical and Applied Genetics 109: 1188-1195.

[32] Maughan, P. J., Smith, S. M., Rojas-Beltran, J. A., Elzinga, D., Raney, J. A., Jellen, E. N., Bonifacio, A., Udall, J. A., Fairbanks, D. J. (2012): Single nucleotide polymorphism identification, characterization, and linkage mapping in quinoa. - Plant Genome 5: 114125.

[33] Mehmood, A., Jaskani, M. J., Ahmad, S., Ahmad, R. (2013): Evaluation of genetic diversity in open pollinated guava by iPBS primers. - Pakistan Journal of Agricultural Sciences 50: 591-597.

[34] Mehmood, A., Luo, S., Ahmad, N., Dong, C., Mahmood, T., Sajjad, Y., Jaskani, M., Sharp, P. (2016): Molecular variability and phylogenetic relationships of guava (Psidium guajava L.) cultivars using inter-primer binding site (iPBS) and microsatellite (SSR) markers. - Genetic Resources and Crop Evolution 63: 1345-1361.

[35] Mutlu, N., Boyacı, F. H., Göçmen, M., Abak, K. (2008): Development of SRAP, SRAPRGA, RAPD and SCAR markers linked with a Fusarium wilt resistance gene in eggplant. - Theoretical and Applied Genetics 117: 1303.

[36] Nemli, S., Asciogul, T. K., Kaya, H. B., Kahraman, A., Esiyok, D., Tanyolac, B. (2014): Association mapping for five agronomic traits in the common bean (Phaseolus vulgaris L.) - Journal of the Science of Food and Agriculture 94: 3141-3151.

[37] Phong, N. H., Pongnak, W., Soytong, K., Poeaim, S., Poeaim, A. (2016): Diversity of tea (Camellia sinensis (L.) O. Kuntze) grown in Vietnam based on morphological characteristics and inter primer binding sites (iPBS) marker. - International Journal of Agriculture and Biology 18: 385-392.

[38] Prado, R. E., Boero, C., Gallard, M., Gonzalez, J. A. (2000): Effect of $\mathrm{NaCl}$ on germination, growth, and soluble sugar content in Chenopodium quinoa Willd. seeds. Botanical Bulletin- Academia Sinica Taipei 41: 27-34.

[39] Pritchard, J. K., Stephens, M., Donnelly, P. (2000a): Inference of population structure using multilocus genotype data. - Genetics 155: 945-959.

[40] Pritchard, J. K., Stephens, M., Rosenberg, N. A., Donnelly, P. (2000b): Association mapping in structured populations. - The American Journal of Human Genetics 67: 70181.

[41] Rohlf, F. J. (1998): NTSYS-pc. Numerical Taxonomy and Multivariate Analysis System. Version 2. 02. - Exeter Publications, Setauker, NY, USA.

[42] Ruas, P. M., Bonifacio, A., Ruas, C. F., Fairbanks, D. J., Andersen, W. R. (1999): Genetic relationship among 19 accessions of six species of Chenopodium L., by randomly amplified polymorphic DNA fragments (RAPD). - Euphytica 105: 25-32. 
[43] Smykal, P., Bacova-Kerteszova, N., Kalendar, R., Corander, J., Schulman, A. H., Pavelek, M. (2011): Genetic diversity of cultivated flax (Linum usitatissimum L.) germplasm assessed by retrotransposon-based markers. - Theoretical and Applied Genetics 122: 1385-1397.

[44] Ward, S. M. (2000): Allotetraploid segregation for single-gene morphological characters in quinoa (Chenopodium quinoa Willd.). - Euphytica 116: 11-16.

[45] Yeh, F. C., Yang, R. C., Boyle, T. B., Ye, Z., Mao, J. X. (1997): POPGENE, the userfriendly shareware for population genetic analysis. - Molecular Biology and Biotechnology Center, University of Alberta, Canada 10: 295-301.

[46] Yildı, M., Koçak, M., Baloch, F. (2015): Genetic bottlenecks in Turkish okra germplasm and utility of iPBS retrotransposon markers for genetic diversity assessment. - Genetics and Molecular Biology 14: 10588-10602.

[47] Yoon, M. Y., Moe, K. T., Kim, D. Y., Rho, I. R., Kim, S., Kim, K. T., Park, Y. J. (2012): Genetic diversity and population structure analysis of strawberry (Fragaria $\mathrm{x}$ ananassa Duch.) using SSR markers. - Electronic Journal of Biotechnology 15: 6-6.

[48] Zargar, S. M., Sharma, A., Sadhu, A., Agrawal, G. K., Rakwal, R. (2014): Exploring genetic diversity in common bean from unexploited regions of Jammu and KashmirIndia. - Molecular Plant Breeding 5: 5-9.

[49] Zargar, S. M., Farhat, S., Mahajan, R., Bhakhri, A., Sharma, A. (2016): Unraveling the efficiency of RAPD and SSR markers in diversity analysis and population structure estimation in common bean. - Saudi Journal of Biological Sciences 23: 39-149.

[50] Zeinalzadehtabrizi, H., Hosseinpour, A., Aydin, M., Haliloglu, K. (2015): A modified genomic DNA extraction method from leaves of sunflower for PCR based analyzes. Journal of Biodiversity and Environmental Sciences 7: 222-225.

[51] Zhang, T., Gu, M., Liu, Y., Lv, Y., Zhou, L., Lu, H., Zhao, H. (2017): Development of novel InDel markers and genetic diversity in Chenopodium quinoa through wholegenome re-sequencing. - BMC Genomics 18: 685. 